\title{
COBIE-BASED LIGHTWEIGHT REPRESENTATION OF A BUILDING NAVIGATION NETWORK
}

\author{
Saurabh Taneja $^{1 *}$, Asli Akcamete ${ }^{1}$, Burcu Akinci ${ }^{1}$, James H. Garrett ${ }^{1}$, Jr., Lucio Soibelman ${ }^{1}$, and E. William East ${ }^{2}$ \\ ${ }^{1}$ Department of Civil and Environmental Engineering, Carnegie Mellon University, Pittsburgh, USA \\ ${ }^{2}$ CERL, Engineer Research and Development Center, Chicago, USA \\ *Corresponding author (staneja@andrew.cmu.edu)
}

\begin{abstract}
A navigation network is required for analysis in several activities during various phases of a building lifecycle, such as building accessibility analysis [22], building egress code compliance ([15], [11]), wayfinding [10], indoor positioning [18] and navigation guidance [18]. Various models for representing navigation networks have been proposed in literature [1], such as graph-based, set-based and hybrid models, but there is lack of a standard representation for exchanging navigation network information. We have identified the information requirements for representing navigation networks in AEC domain, such as spatial connectivity, network distance and network geometry, to name a few. The identified requirements have been mapped to existing navigation network representations, such as graph-based, set-based and hybrid models, to create a lightweight representation of navigation networks in the Construction Operations Building Information exchange (COBie) schema [4]. Our developed lightweight representation is based on a hybrid model and involves extending the COBie schema with 'Node', 'Edge' and 'Node-Tree' tables and utilizing the existing 'Coordinate' table in the COBie schema. We conclude this paper with a discussion on the implementation of the proposed representation of navigation networks in the COBie schema.
\end{abstract}

Keywords: Geometric Topology Network, Navigation guidance, COBie, BIM

\section{INTRODUCTION}

In the Architecture, Engineering and Construction (AEC) domain, various applications require automated navigation network analysis such as building accessibility analysis [22], building egress code compliance analysis ([15], [11]), wayfinding [10] and indoor positioning [18]. Currently, there is no available standard for exchanging navigation network information within facilities. With a growing demand for providing automated navigation guidance and positioning support for elderly, physically challenged or other users in need, there is a necessity to exchange navigation network information with ubiquitous mobile devices that can assist mobile users. Although Industry Foundation Classes (IFC)-based Building Information Model (BIM) files contain an implicit representation of the building topology through ifcRelationship classes, BIM files require numerous computations to derive the explicit navigation network of a building.
Construction Operations and Building Information Exchange (COBie) standard allows retrieving process specific IFC-based facility data but uses spreadsheets, a lightweight representation, to view and store this data [4]. Due to the fact that COBie schema is lightweight and hence can be easily exchanged, we have selected it to develop a representation for navigation networks in indoor environments. The representation has been developed using a set of requirements identified for automated analysis of navigation networks in different application domains and a mapping of existing representations based on the identified requirements.

The next section presents an overview of the COBie standard. Section 3 details the requirements for navigation network analysis. Section 4 describes the research approach, including literature review and the representation of the developed building geometric topology network. Section 5 concludes this research paper. 


\section{CONSTRUCTION OPERATIONS AND BUILDING INFORMATION EXCHANGE (COBie) STANDARD}

Construction Operations Building Information Exchange (COBie) is a specification that streamlines the capture of handover information that is essential to support operation, management, and maintenance of the facilities. COBie contains worksheets for lightweight transfer of specific building information that is retrieved from IFC files. COBie resembles a relational database structure as opposed to the object-oriented structure of an IFC file [4]. There are different worksheets in COBie schema, such as Facility, Floor, Space, Zone and Coordinate, which contain specific attributes [4]. These worksheets can be utilized, extended and new worksheets can be created to represent navigation networks in indoor environments.

\section{REQUIREMENTS FOR NAVIGATION NETWORK ANALYSIS}

This section details the requirements for analysis of navigation networks in different applications in the AEC domain.

\subsection{Building accessibility analysis}

Building accessibility analysis involves determining whether all people irrespective of any disability, including those with physical, sensory and cognitive impairments, can access the building. As shown in Table 1, accessibility analysis requires knowledge of spatial connectivity, width of portals, portal type (door, elevator or stair) and floor elevation.

\subsection{Building egress code compliance checking}

A number of building codes in various countries require the building design, construction and layout to confirm with rules and provisions regarding unobstructed human circulation from anywhere in the building to egress doors. Building egress code compliance checking requires spatial connectivity, network distance and portal type (egress door) as shown in Table 1.

\subsection{Indoor Wayfinding}

Wayfinding is the ability to determine the route from point A to point B in an indoor environment. Wayfinding essentially requires the knowledge of spatial connectivity and network distance to find the shortest route (Table 1).

\subsection{Indoor-positioning}

Indoor positioning is the process of determining the position of a user or an object in an indoor environment automatically. Due to a number of drawbacks that various ubiquitous and low-cost indoor positioning technologies suffer from, there is a need for navigation networks to correct the erroneous positioning data ([18], [6]). For this purpose, navigation networks should accurately represent both the topology and the geometry of the navigation paths ([18], [19]). Therefore network analysis for indoor positioning requires network geometry in addition to spatial connectivity and network distance (Table 1).

\subsection{Indoor navigation guidance}

The process of providing automated turn-by-turn directions when moving within environments is called navigation guidance. Network analysis for navigation guidance requires knowledge of network geometry, portal type (stair or elevator or door) and floor elevation to provide users with turn-by-turn guidance (Table 1)

Table 1. Information requirements for navigation network analysis in different application domains

\begin{tabular}{|c|c|c|c|c|c|c|}
\hline $\begin{array}{l}\text { Information } \\
\text { requirements } \\
\text { for navigation } \\
\text { network } \\
\text { analysis --> }\end{array}$ & 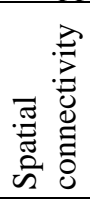 & 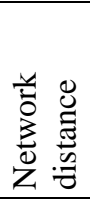 &  & 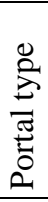 & 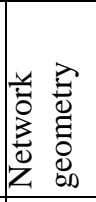 & 它 \\
\hline $\begin{array}{l}\text { Building } \\
\text { accessibility } \\
\text { analysis } \\
\end{array}$ & $\mathrm{X}$ & & $\mathrm{X}$ & $\mathrm{X}$ & & $\mathrm{X}$ \\
\hline $\begin{array}{l}\text { Egress code } \\
\text { compliance } \\
\text { checking }\end{array}$ & $\mathrm{X}$ & $\mathrm{X}$ & & $\mathrm{X}$ & & \\
\hline Wayfinding & $\mathrm{X}$ & $\mathrm{X}$ & & & & \\
\hline $\begin{array}{l}\text { Indoor } \\
\text { positioning }\end{array}$ & $\mathrm{X}$ & $\mathrm{X}$ & & & $\mathrm{X}$ & $\mathrm{X}$ \\
\hline $\begin{array}{l}\text { Indoor } \\
\text { navigation } \\
\text { guidance } \\
\end{array}$ & $\mathrm{X}$ & $\mathrm{X}$ & & $\mathrm{X}$ & $\mathrm{X}$ & $\mathrm{X}$ \\
\hline
\end{tabular}

\section{CATEGORIZATION AND MAPPING OF EXISTING NAVIGATION NETWORK REPRESENTATIONS}

A number of researchers in the pervasive computing domain have developed location models to represent the spatial interactions in indoor environments. These location models represent the navigation network of the concerned 
indoor environments by virtue of modeling the spatial interactions such as 'connected to' relationships between different spaces. Becker et al. [1] have reviewed the existing location models developed by various researchers and categorized them into five broad classes, namely, setbased, hierarchical, graph-based, combined symbolic and hybrid models. The first four categories of location-models deal with symbolic location data, such as rooms, corridors and sub-spaces. Hybrid models deal with both geometric coordinates and symbolic coordinates.

Set-based location models involve representing spatial entities as sets of smaller entities. For example, a floor can be represented as a set of all the rooms contained in it. Similarly, a building can be represented as a set of all the floors. Set-based location models also include 'connected to' sets that contain spatial entities connected to a particular space. For example, the 'connected to' set of a particular space will contain all the spaces that are connected to the concerned space. Hierarchical models create a hierarchy of spaces, such as buildings, floors, wings and spaces, but do not necessarily represent the spatial connections. These models are useful for aggregating or disaggregating spatial data using the hierarchies, but are not used for describing the navigation network in indoor environments.

Graph-based models have been most widely used for describing navigation networks ([2]). These models consist of 'nodes' and 'edges' in a graph network or a 'node-tree'. Graph-based models are symbolic models, where 'nodes' represent symbolic coordinates, such as rooms, and the 'edges' represent the physical connections between these nodes. Additional information can be embedded in the 'edges' to represent the network distance or the properties of the connection. Combined symbolic models are graphmodels, but they also contain sets of 'nodes', similar to hierarchical models, that lie at the same level in the hierarchy. Hence, combined symbolic models can be used to represent navigation networks as well as aggregate and disaggregate spatial data. Hybrid models are similar to graph-based models and represent both geometric and symbolic spaces. In hybrid models the 'nodes' can have geometric coordinates. Due to the fact that a hybrid model contains geometric coordinates, it resembles the skeleton network structure of an indoor environment. Hybrid models are useful for geometric analysis, such as range and nearest neighbor queries.

In the AEC domain, Roth and Hashimshony [17] and Wu et al. [22] utilized a graph-model for accessibility analysis. Roth and Hashimshony [17] had proposed a graph-based representation for addressing architectural problems, which $\mathrm{Wu}$ et al. [22] adopted and utilized the IFC-based BIM files for automated generation of the accessibility network of the indoor environment. They represented rooms as nodes in the graph network and the connections between rooms as 'edges'. Han et al. [7] and Pruski [16] both utilized geometric representations for running simulations of wheelchair movement in indoor environments that require detailed geometric information.

Nassar and Nguyen [15] developed a means of egress code compliance checking (MECC) representation. MECC resembles a graph-based representation as it contains provision for representing spaces and connections just like a graph network. Kannala [9] utilized a metric model that utilizes the whole geometric representation of the indoor environment for calculating escape route distances. Lee et al. [11] developed a hybrid model based circulation network for egress distance code compliance checking. The circulation network consists of geometric coordinates as nodes that correspond to decision points or points where a user deviates from a straight line.

Researchers have utilized both graph-based models and hybrid models for addressing wayfinding ([10], [21]) and navigation guidance ([1], [12], [14]) in indoor environments. The main difference in using a graph-based model versus a hybrid model is the fact that researchers have represented the geometric information, such as the network distance, as an attribute of the 'edge' in the graphbased model. Applications involving indoor positioning mainly require geometric or hybrid models for correcting erroneous positioning data. There is a need to represent explicit geometry of the nodes and the edges for correcting positioning data [18]. Table 2 presents the categorization of the existing models for network representation and the mapping of selected literature to the existing models. We have omitted hierarchical models and combined models 
from the categorization proposed by Becker et al. [1] as hierarchical models are not suited for navigation network representation and combined models resemble graph-based models for navigation network representation.

Table 2. Mapping of navigation network analysis literature to existing categorization of navigation network models

\begin{tabular}{|l|l|l|l|l|}
\hline & $\begin{array}{l}\text { Set- } \\
\text { based }\end{array}$ & $\begin{array}{l}\text { Graph- } \\
\text { based }\end{array}$ & $\begin{array}{l}\text { Hybrid } \\
\text { model }\end{array}$ & $\begin{array}{l}\text { Geometric } \\
\text { model }\end{array}$ \\
\hline $\begin{array}{l}\text { Building } \\
\text { accessibility } \\
\text { analysis }\end{array}$ & & $\begin{array}{l}{[17],} \\
{[22]}\end{array}$ & & {$[7],[16]$} \\
\hline $\begin{array}{l}\text { Egress code } \\
\text { compliance } \\
\text { checking }\end{array}$ & & $\begin{array}{l}{[15],} \\
{[23]}\end{array}$ & {$[11]$} & {$[9]$} \\
\hline Wayfinding & & {$[21]$} & {$[10]$} & \\
\hline $\begin{array}{l}\text { Indoor } \\
\text { positioning }\end{array}$ & {$[20]$} & {$[8]$} & {$[18]$,} & {$[13]$} \\
\hline $\begin{array}{l}\text { Indoor } \\
\text { navigation } \\
\text { guidance }\end{array}$ & {$[3]$} & {$[14]$} & {$[1],[12]$} & \\
\hline
\end{tabular}

\section{REPRESENTATION OF THE BUILDING NAVIGATION NETWORK}

It is clear from the requirements analysis, literature review and mapping that graph-based and hybrid models are better suited for representing navigation networks as they support majority of applications. Hybrid models enjoy the advantage of representing navigation network geometry over symbolic graph-based models. For example, in building accessibility analysis, a graph-based symbolic representation of indoor environment can depict which spaces are all-accessible, but for detailed simulation of wheelchair movement, geometry of indoor environments has to be modeled. A hybrid-model also suits egress code compliance checking as it accurately represents the escape route distances by virtue of capturing navigation path geometry [11]. Hence, we have selected a hybrid model for representing the navigation networks in indoor environments. We refer to a navigation network based on a hybrid model as a Geometric Topology Network (GTN), because a hybrid model depicts geometry of the network. The developed COBie-based lightweight representation of the GTN contains four main tables, namely 'Node', 'Coordinate', 'Edge’ and 'Node-Tree'.
'Node' is a decision point or a point where a user deviates from a straight path in the network. A 'node' can represent a point in a room or a door or other portals, such as an egress door, a stair or an elevator. Node table (Table 3) contains five required attributes, namely NodeID, CoordinateID of the coordinate that forms the node, NodeType, ObjectID of the building element the node represents and NodeTreeID of the node-tree to which the node belongs. CoordinateID is a foreign key from 'Node' table to 'Coordinate' table. ObjectID is required to carry out analysis on the corresponding object, for example, the door width or floor finish height for accessibility analysis. The universally unique identifier (UUID) standard represents all the identifiers, such as NodeID or ObjectID.

Table 3. Proposed 'Node' table in COBie standard

\begin{tabular}{|l|l|l|l|}
\hline Name & Type & Reqd & Description \\
\hline NodeID & String & Reqd & UUID of the node \\
\hline CoordinateID & String & Reqd & $\begin{array}{l}\text { UUID of the related } \\
\text { coordinate }\end{array}$ \\
\hline NodeType & String & Reqd & $\begin{array}{l}\text { Enum: Space point, } \\
\text { Internal door, External } \\
\text { door, Elevator, Stair }\end{array}$ \\
\hline NodeTreeID & String & Reqd & $\begin{array}{l}\text { UUID of the related } \\
\text { building element } \\
\text { NodeTree }\end{array}$ \\
\hline
\end{tabular}

'Coordinate' table (Table 4) represents geometric or symbolic coordinates. This table contains two required attributes, namely CoordinateID and CoordinateType. CoordinateType defines whether the coordinate is a geometric or symbolic coordinate. A geometric coordinate can have $\mathrm{x}, \mathrm{y}$ and $\mathrm{z}$ values, whereas a symbolic coordinate will represent a space (representation format to be determined). Geometric representation enables calculation of network distances, as well as provision of turn-by-turn navigation. For applications that do not require geometric information, symbolic coordinates will suffice. A 'Node' table is different from a 'Coordinate' table because the former represents properties of a semantic node (space, door, elevator) in the graph and the latter represents the physical realization of the semantic node. Moreover, 'Coordinate' is an existing worksheet in COBie format that can be referred to from the 'Node' table.

Table 4. Proposed 'Coordinate' table in COBie standard 


\begin{tabular}{|l|l|l|l|}
\hline Name & Type & Reqd & Description \\
\hline CoordinateID & String & Reqd & $\begin{array}{l}\text { UUID of the } \\
\text { coordinate }\end{array}$ \\
\hline $\begin{array}{l}\text { Coordinate- } \\
\text { Type }\end{array}$ & String & Reqd & $\begin{array}{l}\text { Enum: Geometric } \\
\text { or Symbolic }\end{array}$ \\
\hline CoordinateX & Number & Opt & $\begin{array}{l}\text { Abscissa of the } \\
\text { coordinate }\end{array}$ \\
\hline CoordinateY & Number & Opt & $\begin{array}{l}\text { Ordinate of the } \\
\text { coordinate }\end{array}$ \\
\hline CoordinateZ & Number & Opt & $\begin{array}{l}\text { Height of the } \\
\text { coordinate }\end{array}$ \\
\hline $\begin{array}{l}\text { Coordinate- } \\
\text { Location }\end{array}$ & Complex & Opt & $\begin{array}{l}\text { Symbolic } \\
\text { coordinate, format } \\
\text { to be determined }\end{array}$ \\
\hline
\end{tabular}

The 'Edge' table (Table 5) contains five required attributes, namely, EdgeID, EdgeType, FirstNodeID, SecondNodeID and isBidirectional. EdgeType can be horizontal if it connects two nodes at same building level, or vertical if it is an inter-level connection. The attribute isBidirectional is a boolean that states whether the edge is bidirectional or not. This attribute can represent portals that require authorization. In cases where an edge is not bidirectional, the flow is from the first node to the second.

Table 5. Proposed 'Edge' table in COBie standard

\begin{tabular}{|l|l|l|l|}
\hline Name & Type & Reqd & Description \\
\hline EdgeID & String & Reqd & UUID of the edge \\
\hline EdgeType & String & Reqd & $\begin{array}{l}\text { Enum: Horizontal } \\
\text { or vertical edge }\end{array}$ \\
\hline FirstNodeID & String & Reqd & $\begin{array}{l}\text { UUID of the first } \\
\text { node }\end{array}$ \\
\hline SecondNodeID & String & Reqd & $\begin{array}{l}\text { UUID of the } \\
\text { second node }\end{array}$ \\
\hline isBidirectional & Boolean & Reqd & $\begin{array}{l}\text { Bidirectional edge } \\
\text { or not }\end{array}$ \\
\hline
\end{tabular}

'Node-Tree' is the graph that contains the nodes and the edges. The 'Node-Tree' table (Table 6) contains two required attributes, namely NodeTreeType, which can be a building, floor or wing node-tree, and NodeTreeID. Depending upon the type of node-tree, we can have several optional attributes, such as elevation of the floor node-tree. Every table in the proposed representation can be extended based upon different requirements for analysis in different domains. For example, if a wayfinding application has to be extended for allowing multiple queries, such as, finding the shortest route that contains maximum number of windows, then the 'node' table can be extended to represent the number of windows in a space if the value of NodeType attribute is a space.

Table 6. Proposed 'Node-Tree' table in COBie standard

\begin{tabular}{|l|l|l|l|}
\hline Name & Type & Reqd & Description \\
\hline NodeTreeID & String & Reqd & $\begin{array}{l}\text { UUID of the node- } \\
\text { tree }\end{array}$ \\
\hline NodeTreeType & String & Reqd & $\begin{array}{l}\text { Enum: Building, } \\
\text { Floor, Wing, etc. }\end{array}$ \\
\hline BuildingID & String & Opt & $\begin{array}{l}\text { Elevation of the } \\
\text { floor node-tree }\end{array}$ \\
\hline WingName & String & Opt & $\begin{array}{l}\text { Nume of the } \\
\text { related building }\end{array}$ \\
\hline
\end{tabular}

We have implemented the proposed representation of a navigation network in a prototype for correcting indoor positioning data. Implementation of a prototype to evaluate the exchange of this navigation network representation with mobile devices is planned for the near future.

\section{CONCLUSIONS AND DISCUSSIONS}

In this paper, we have presented a lightweight representation of a navigation network based on the COBie standard. A hybrid location model is selected for modeling the navigation network based on literature review, categorization of existing approaches and requirement identification for navigation network analysis. The developed representation contains four tables, namely, 'node', 'coordinate', 'edge' and 'node-tree'.

The proposed representation can be implemented in COBie standard by creating three new worksheets, namely 'Node', 'Edge' and 'Node-Tree'. Each worksheet will contain the attributes listed in Tables 3, 5 and 6 respectively. Each worksheet will also have attributes required by COBie schema such as Name, CreatedBy, CreatedOn and Category. 'Coordinate' is an existing table in COBIe schema that already contains CoordinateX, CoordinateY and CoordinateZ attributes. This table can be extended to represent CoordinateType and then referred from 'Node' table using the CoordinateID.

\section{ACKNOWLEDGEMENTS}

The work presented in this paper is funded by Engineer Research and Development Center (ERDC) of the US 
Army Corps of Engineers (USACE). All support is gratefully acknowledged.

\section{REFERENCES}

[1] Becker, T., C. Nagel, and T. H. Kolbe, “A multilayered Space-Event model for navigation in indoor spaces", In Lecture Notes in Geoinformation and Cartography, Chapter 5, pp. 61-77. Springer Berlin Heidelberg, 2009.

[2] Bollobas, B., “Modern Graph Theory”, Springer, 1998.

[3] Cheverst K., N. Davies, K. Mitchell, A. Friday, "Experiences of developing and deploying a context-aware tourist guide: the GUIDE project”, MobiCom 2000, Boston, pp 20-31, 2000.

[4] East, E. W., "Construction Operations Building Information Exchange: Requirements Definition and Pilot Implementation Standard”, Technical Report USACE, ERDC/CERL TR-07-03, 2007.

[5] Ferris, B., D. Hähnel, D. Fox, “Gaussian Processes for Signal Strength-Based Location Estimation”, In Proceedings of Robotics Science and Systems, 2006.

[6] Glanzer, G., T. Bernoulli, T. Wiessflecker, U. Walder, "Semi-autonomous indoor positioning using MEMS-based inertial measurement units and building information," 6th Workshop on Positioning, Navigation and Communication, pp 135-139, 2009.

[7] Han, C.S., J.C. Kunz and K.H. Law, "Compliance Analysis for Disabled Access”, Advances in Digital Government, p. 149-163, 2002.

[8] Jensen, C. S., H. Lu, and B. Yang, “Graph model based indoor tracking”, Mobile Data Management, 2009.

[9] Kannala M., ' Escape route analysis based on building information models: design and implementation", MSc thesis, University of Technology, Helsinki, 2005.

[10] Lee, J., “A Spatial Access-Oriented Implementation of a 3-D GIS Topological Data Model for Urban Entities”, GeoInformatica 8 (3), 237-264, 2004.

[11] Lee, J.-k., C. M. Eastman, J. Lee, M. Kannala, and Y.s. Jeong, "Computing walking distances within buildings using the universal circulation network”, Environment and Planning B: Planning and Design, 37 (4), 628-645, 2010.
[12] Lertlakkhanakul, J., Y. Li, J. Choi, and S. Bu, "GongPath: Development of BIM based indoor pedestrian navigation system”, In NCM '09, Washington, DC, USA, pp. 382-388, 2009.

[13] Liao, L., D. Fox, J. Hightower, H. Kautz, and D. Schulz, "Voronoi tracking: location estimation using sparse and noisy sensor data”, In Intelligent Robots and Systems, pp. 723-728, 2003.

[14] Lorenz, B., H. Ohlbach, and E.-P. Stoffel, “A hybrid spatial model for representing indoor environments”, In v 4295 of LNCS, Chapter 10, pp. 102-112. Springer, 2006.

[15] Nassar, K. and T. H. Nguyen, "Using building topological information to check for means of egress building code compliance”, ITcon, 10, 219-227, 2005.

[16] Pruski, A., “A unified approach to accessibility for a person in a wheelchair”, Robotics and Autonomous Systems 58, 1177-1184, 2010.

[17] Roth, J. and R. Hashimshony, "Algorithms in graph theory and their use for solving problems in architectural design”, Computer-Aided Design 20 (7), 373-381, 1988.

[18] Spassov, I., “Algorithms for Map-Aided Autonomous Indoor Pedestrian Positioning and Navigation”, Doctoral Thesis, Ecole polytechnique fédérale de Lausanne, 2007. [19] Taneja, S., B. Akinci, J.H. Garrett, Jr., E.W. East and L. Soibelman, “Transforming an IFC-based Building Layout Information into a Geometric Network Model for Indoor Navigation Assistance,” ASCE Workshop on Computing in Civil Engineering, Miami, 19-22 June, 2011.

[20] Want R., A. Hopper, J. Gibbons, "The active badge location system”, ACM Trans Inf Syst 10:91-102, 1992

[21] Werner, S., B. Krieg-Brückner, and T. Herrmann, "Modelling navigational knowledge by route graphs", in Spatial Cognition II LNAI 1849295 -316, 2000.

[22] Wu, S., A. Lee, W. W. I. Koh, G. Aouad, and C. Fu, "An IFC-based space analysis for building accessibility layout for all users”, Construction Innovation: Information, Process, Management 4 (3), 129-141, 2004.

[23] Yang, Q., “Design knowledge modeling and software implementation for building code compliance checking", Building and Environment 39 (6), 689-698, 2004. 